\title{
MORTALIDAD POR LESIONES DE TRÁNSITO EN CALI COLOMBIA
}

Pablo Chaparro Narváez Instituto Nacional de Salud Colombia

Willian León Quevedo Instituto Nacional de Salud Colombia

Carlos Castañeda Orjuela Instituto Nacional de Salud Colombia 
Panorama Económico, Vol. 27 - No. 3 (Julio - Septiembre de 2019), pp. 701-720

Pablo Chaparro Narváez

Willian León Quevedo

Carlos Castañeda Orjuela

\title{
Mortalidad por lesiones de tránsito en Cali Colombia
}

\section{Resumen}

Las lesiones de tránsito son un importante problema de salud pública por la discapacidad y muerte que producen y la carga económica que generan. El objetivo del estudio fue identificar las características y patrones de distribución espacial y temporal de las muertes debidas a lesiones de tránsito en Cali. Se realizó un estudio descriptivo con base en la información de mortalidad que produce el Departamento Administrativo Nacional de Estadística (DANE). Una frecuencia alta de defunciones se presentó en las comunas 3, 8, 9 y 19. La mayor parte de las muertes se presentaron entre motociclistas y peatones. La tasa promedio de mortalidad para el periodo (2008-2013) fue de 13,6 fallecimientos por 100.000 habitantes. Temporal y espacialmente, la mortalidad evidenció disminución durante el periodo estudiado y se identificaron agrupamientos en distintas comunas del municipio. Estos resultados pueden contribuir en la prevención de las lesiones y de la mortalidad, así como de la promoción de la seguridad vial desde distintos sectores sociales y en particular sobre los actores viales involucrados.

Palabras clave: Mortalidad, lesiones de tránsito, sistemas de información geográfica, análisis espacial

\section{Mortalité pour les blessures de transit en Cali Colombie}

\section{Résumé:}

Les accidents de la circulation sont un problème de santé publique important en raison de l'invalidité et des décès qu'ils provoquent et du fardeau économique qu'ils génèrent. L'objectif de l'étude était d'identifier les caractéristiques et les modèles de distribution spatiale et temporelle des décès dus aux accidents de la circulation à Cali (Colombie) entre 2008 et 2013. Une étude descriptive a été réalisée sur la base des informations sur la mortalité produites par l'Institut national colombien de statistique. Une fréquence élevée de décès s'est produite dans les communes 3, 8, 9 et 19. La plupart des décès sont survenus entre des motocyclistes et des piétons. Le taux de mortalité moyen pour la période était de 13,6 décès pour 100000 habitants. Temporairement et spatialement, la mortalité a montré une baisse au cours de la période étudiée et des regroupements ont été identifiés dans différentes communes de la commune. Ces résultats peuvent contribuer à la prévention des blessures et de la mortalité, ainsi qu'à la promotion de la sécurité routière dans différents secteurs sociaux et, en particulier, les acteurs de la route impliqués.

Mots clés: Mortalité, blessures de la route, systèmes d'information géographique, analyse spatiale

\section{Mortality for transit injury in Cali Colombia}

\begin{abstract}
Traffic injuries are an important public health problem due to the disability and death they cause and the economic burden they generate. The objective of the study was to identify the characteristics and patterns of spatial and temporal distribution of deaths due to traffic injuries in Cali (Colombia) during 2008 - 2013. A descriptive study was carried out based on the mortality information produced by the Colombian National Statistics Institute. A high frequency of deaths occurred in communes 3, 8, 9 and 19. Most of the deaths occurred between motorcyclists and pedestrians. The average mortality rate for the period was 13.6 deaths per 100,000 inhabitants. Temporary and spatially, the mortality showed a decrease during the period studied and groupings were identified in different communes of the municipality. These results can contribute to the prevention of injuries and mortality, as well as the promotion of road safety from different social sectors and, in particular, the road actors involved.
\end{abstract}

Keywords: Mortality, traffic injuries, geographic information systems, spatial analysis 


\section{Mortalidad por lesiones de tránsito en Colombia}

INFORMACIÓN DEL ARTíCULO

Recepción del artículo: 14/02/2019

Concepto de evaluación: 30/03/2019

Aceptación del artículo: 15/06/2019
Pablo Chaparro Narváez Instituto Nacional de Salud, Colombia

Willian León Quevedo Instituto Nacional de Salud, Colombia

Carlos Castañeda-Orjuela Instituto Nacional de Salud, Colombia

\section{INTRODUCCIÓN}

Las lesiones de tránsito son un importante problema de salud pública por la discapacidad y muerte que producen y la carga económica que generan. Estas lesiones se presentan como consecuencia del rápido crecimiento económico y el aumento de los vehículos de motor en los años recientes. Para 2010, la Organización Mundial de la Salud (OMS) reportó 1,24 millones de muertes en el mundo debidas a lesiones de tránsito, que afectaron principalmente a las personas de edades comprendidas entre los 15 y los 44 años (World Health Organization, 2013). Para las Américas se estimaron 153.789 muertes (15,9 muertes por 100.000 habitantes) y para Colombia 8.107 (16,8 por 100.000 habitantes) (World Health Organization, 2015). En Colombia, dentro del grupo de las muertes debidas a causas externas, las lesiones de tránsito ocuparon la segunda posición y en 2014 fueron responsables del 6.402 de defunciones, en las que el 80,5\% fueron hombres y el 57,0\% de los fallecidos estaban entre los 15 y los 44 años (Instituto Nacional de Medicina Legal y Ciencias Forenses, 2015).

En Cali, la tercer ciudad más grande de Colombia, de acuerdo con las cifras del Instituto Nacional de Medicina Legal y Ciencias Forenses, en 2015 se presentaron 434 fallecimientos consecuencia de lesiones de tránsito (Instituto Nacional de Medicina Legal y Ciencias Forenses, 2016). En el periodo de 2005-2014 murieron 3.379 personas. Es posible que estas defunciones sean el resultado del aumento de los límites de velocidad (de 60 a 80 kilómetros por hora en la autopistas Simón Bolívar y Sur-oriental) y la ampliación del horario de la Ley Zanahoria (que pasó de las 3:00 a.m. a 4:00 a.m. de jueves a sábado) (Cuervo, 2016; Instituto Nacional de Medicina Legal y Ciencias Forenses, 2016). 
La discapacidad y muerte causadas por lesiones de tránsito son predecibles y prevenibles. Para ayudar en su reducción, la legislación sobre seguridad vial ha contribuido a mejorar el comportamiento de los usuarios de las vías de tránsito y ha permitido disminuir las lesiones en las vías, y los traumatismos y muertes derivados de estos. La legislación también ha hecho énfasis en cinco factores de riesgo que afectan a la seguridad vial: la velocidad, la conducción bajo los efectos del alcohol, el uso y la calidad del casco cuando se circula en motocicleta, el uso del cinturón de seguridad y el uso de sistemas de retención infantil (Instituto Nacional de Medicina Legal y Ciencias Forenses, 2015).

En marzo de 2010, la Asamblea General de las Naciones Unidas proclamó el "Decenio de Acción para la Seguridad Vial" para el periodo 2011-2020, con el objetivo de estabilizar y reducir las cifras previstas de víctimas mortales en lesiones de tránsito en todo el mundo, aumentando las actividades en los niveles nacional, regional y mundial (Naciones Unidas, 2010). En concordancia con esta Asamblea, Colombia, diseñó el "Plan Nacional de Seguridad Vial 2011 - 2021" que estableció como objetivo reducir el número de víctimas fatales en un 26\% por lesiones de tránsito a nivel nacional para 2021 (Colombia, 2015). Recientemente, una de las metas de los Objetivos de Desarrollo Sostenible (ODS) ha sido que para 2020, se reduzca a la mitad el número de muertes y lesiones por accidentes de tráfico en el mundo (Programa de las Naciones Unidas para el desarrollo, 2015).

En Colombia distintos estudios han permitido conocer la frecuencia, distribución y factores de riesgo asociados a las lesiones de tránsito. Anualmente se emiten informes sobre el comportamiento de los casos y muertes debidos a lesiones de tránsito (Instituto Nacional de Medicina Legal y Ciencias Forenses, 2015), e informes no periódicos, realizan el seguimiento a las estadísticas de casos y muertes por lesiones de tránsito (Colombia. Contraloría General de la República, 2012, 2014). En Cali, un estudio sobre actitudes y comportamientos de los peatones en los sitios de alta accidentalidad concluyó que la adecuación y mantenimiento de la infraestructura vial, la construcción de nuevos instrumentos viales y la recuperación del espacio público no eran suficientes para la prevención de las lesiones de tránsito, sino que se debía concientizar y educar a los conductores y peatones para lograr modificar ciertos comportamientos y actitudes erradas que se presentaban en el sistema vial (Echeverry, Mera, Villota, \& Zárate, 2005). Otro estudio en este municipio, que investigaba la asociación entre la implementación de políticas de control de alcohol y cambios en la incidencia de muertes por lesiones de tránsito, mostró que las políticas de control de alcohol más restrictivas reducían la mortalidad (Sánchez, Suffoleto, Mena, Gutiérrez, \& Puyana, 2014). Adicionalmente, el análisis de la mortalidad ocasionada por vehículos de motor en las personas mayores de 60 años en Cali, indicó que estaban comprometidos factores relacionados con el agente (conductor y tipo de vehículo), el huésped (actitud del conductor al conducir, consumo de licor por el conductor, alta velocidad usada en los vehículos, cultura del predominio del vehículo sobre el peatón) y el medio (señales de tránsito, diseño arquitectónico y vías de la ciudad) (Reyes-Ortiz, Espitia, Vélez, \& Espinoza, 1998).

El estudio de la mortalidad debida a lesiones de tránsito, en los últimos años se ha apoyado de los sistemas de información geográfica (SIG), que se han convertido en una herramienta valiosa para la investigación epidemiológica (Dummer, 2008). En Cali son pocas las investigaciones sobre mortalidad debida a lesiones de tránsito que han utilizado los SIG para analizar las ubicaciones de las lesiones viales (Olaya, 2015). El objetivo de este estudio fue identificar las características y los patrones de distribución espacial y temporal de las muertes debidas a lesiones de tránsito que ocurrieron en Cali (Valle del Cauca) entre 2008 y 2013. 


\section{MÉTODO}

Se realizó un estudio descriptivo para identificar las características y los patrones de distribución temporal y espacial de las muertes debidas a lesiones de tránsito que ocurrieron en Cali entre 2008 y 2013.

Cali, la capital del departamento del Valle del Cauca, está situada al occidente del río Cauca, en el suroeste de Colombia. Tiene una extensión territorial de 564 km2 (21,2\% urbano). En 2013 la población estimaba ascendía a 2.319.655 habitantes distribuidos en 22 comunas y 15 corregimientos. La densidad demográfica era de 41,4 habitantes por km2. El índice de desarrollo humano (IDH) en 2005 fue de 0,802.

Se estudiaron las muertes codificadas como lesiones de tránsito de acuerdo con las normas establecidas por la Clasificación Internacional de Enfermedades - décima revisión (CIE10) (Organización Panamericana de la Salud, 1995) (Cuadro 1).

\section{Cuadro 1.}

Códigos para muertes debidas a lesiones de transito

\begin{tabular}{|c|c|c|}
\hline Categoría & A tres caracteres & A cuatro caracteres \\
\hline $\begin{array}{l}\text { Peatón lesionado en } \\
\text { accidente de transporte }\end{array}$ & V01-V04, V09 & V01.1, V02.1, V03.1, V04.1, V09.2, V09.3 \\
\hline $\begin{array}{l}\text { Ciclista lesionado en } \\
\text { accidente de transporte }\end{array}$ & V10-V19 & $\begin{array}{l}\text { V10.4, V10.5, V10.9, V11.4, V11.5, V11.9, } \\
\mathrm{V} 12.4, \mathrm{~V} 12.5, \mathrm{~V} 12.9, \mathrm{~V} 13.4, \mathrm{~V} 13.5, \mathrm{~V} 13.9, \\
\mathrm{~V} 14.4, \mathrm{~V} 14.5, \mathrm{~V} 14.9, \mathrm{~V} 15.4, \mathrm{~V} 15.5, \mathrm{~V} 15.9 \\
\mathrm{~V} 16.4, \mathrm{~V} 16.5, \mathrm{~V} 16.9, \mathrm{~V} 17.4, \mathrm{~V} 17.5, \mathrm{~V} 17.9, \\
\mathrm{~V} 18.4, \mathrm{~V} 18.5, \mathrm{~V} 18.9, \mathrm{~V} 19.4, \mathrm{~V} 19.5, \mathrm{~V} 19.6 \\
\mathrm{~V} 19.7, \mathrm{~V} 19.9\end{array}$ \\
\hline $\begin{array}{l}\text { Motociclista lesionado } \\
\text { en accidente de transporte }\end{array}$ & V20-V29 & $\begin{array}{l}\text { V20.4, V20.5, V20.9, V21.4, V21.5, V21.9, } \\
\text { V22.4, V22.5, V22.9, V23.4, V23.5, V23.9, } \\
\text { V24.4, V24.5, V24.9, V25.4, V25.5, V25.9, } \\
\text { V26.4, V26.5, V26.9, V27.4, V27.5, V27.9, } \\
\text { V28.4, V28.5, V28.9, V29.4, V29.5, V29.6, } \\
\text { V29.9 }\end{array}$ \\
\hline $\begin{array}{l}\text { Ocupante de vehículo } \\
\text { de motor de tres ruedas } \\
\text { lesionado en accidente } \\
\text { de transporte }\end{array}$ & V30-V39 & $\begin{array}{l}\text { V30.4, V30.5, V30.9, V31.4, V31.5, V31.9, } \\
\text { V32.4, V32.5, V32.9, V33.4, V33.5, V33.9, } \\
\text { V34.4, V34.5, V34.9, V35.4, V35.5, V35.9, } \\
\text { V36.4, V36.5, V36.9, V37.4, V37.5, V37.9, } \\
\text { V38.4, V38.5, V38.9, V39.4, V39.5, V39.6, } \\
\text { V39.9 }\end{array}$ \\
\hline $\begin{array}{l}\text { Ocupante de automóvil } \\
\text { lesionado en accidente } \\
\text { de transporte }\end{array}$ & V40-V49 & $\begin{array}{l}\text { V40.4, V40.5, V40.9, V41.4, V41.5, V41.9, } \\
\text { V42.4, V42.5, V42.9, V43.4, V43.5, V43.9, } \\
\text { V44.4, V44.5, V44.9, V45.4, V45.5, V45.9, } \\
\text { V46.4, V46.5, V46.9, V47.4, V47.5, V47.9, } \\
\text { V48.4, V48.5, V48.9, V49.4, V49.5, V49.6, } \\
\text { V49.9 }\end{array}$ \\
\hline
\end{tabular}




\section{Cuadro 1.}

Códigos para muertes debidas a lesiones de transito

\begin{tabular}{|c|c|c|}
\hline Categoría & A tres caracteres & A cuatro caracteres \\
\hline $\begin{array}{l}\text { Ocupante de camioneta o } \\
\text { furgoneta lesionado en } \\
\text { accidente de transporte }\end{array}$ & V50-V59 & $\begin{array}{l}\text { V50.4, V50.5, V50.9, V51.4, V51.5, V51.9, } \\
\text { V52.4, V52.5, V52.9, V53.4, V53.5, V53.9, } \\
\text { V54.4, V54.5, V54.9, V55.4, V55.5, V55.9, } \\
\text { V56.4, V56.5, V56.9, V57.4, V57.5, V57.9, } \\
\text { V58.4, V58.5, V58.9, V59.4, V59.5, V59.6, } \\
\text { V59.9 }\end{array}$ \\
\hline $\begin{array}{l}\text { Ocupante de vehículo de } \\
\text { transporte pesado lesionado } \\
\text { en accidente de transporte }\end{array}$ & V60-V69 & $\begin{array}{l}\text { V60.4, V60.5, V60.9, V61.4, V61.5, V61.9, } \\
\text { V62.4, V62.5, V62.9, V63.4, V63.5, V63.9, } \\
\text { V64.4, V64.5, V64.9, V65.4, V65.5, V65.9, } \\
\text { V66.4, V66.5, V66.9, V67.4, V67.5, V67.9, } \\
\text { V68.4, V68.5, V68.9, V69.4, V69.5, V69.6, } \\
\text { V69.9 }\end{array}$ \\
\hline $\begin{array}{l}\text { Ocupante de autobús pesado } \\
\text { lesionado en accidente } \\
\text { de transporte }\end{array}$ & V70-V79 & $\begin{array}{l}\text { V70.4, V70.5, V70.9, V71.4, V71.5, V71.9, } \\
\text { V72.4, V72.5, V72.9, V73.4, V73.5, V73.9, } \\
\text { V74.4, V74.5, V74.9, V75.4, V75.5, V75.9, } \\
\text { V76.4, V76.5, V76.9, V77.4, V77.5, V77.9, } \\
\text { V78.4, V78.5, V78.9, V79.4, V79.5, V79.6, } \\
\text { V79.9 }\end{array}$ \\
\hline $\begin{array}{l}\text { Otras lesiones de } \\
\text { transporte terrestre }\end{array}$ & V83-V87, V89 & $\begin{array}{l}\text { V83.0, V83.1, V83.2, V83.3,V84.0, V84.1, } \\
\text { V84.2, V84.3, V85.0, V85.1, V85.2, V85.3, } \\
\text { V86.0, V86.1, V86.2, V86.3, V87.0-V87.9, } \\
\text { V89.2, V89.3 }\end{array}$ \\
\hline $\begin{array}{l}\text { Secuelas de accidente de } \\
\text { vehículo de motor }\end{array}$ & Y85 & Y85.0 \\
\hline
\end{tabular}

Fuente: Elaboración de los autores partir de Estadísticas vitales, DANE

Se asumió la definición de accidente de tránsito propuesta en la CIE-10. Esta clasificación lo consideró como "cualquier accidente de vehículo que ocurre en la vía pública (por ejemplo, que se origina en, termina en, o afecta parcialmente a un vehículo en la vía pública") (Organización Panamericana de la Salud, 1995). Las fuentes de información empleadas fueron las bases de mortalidad del Departamento Administrativo Nacional de Estadística (DANE) y las proyecciones de población de la misma institución para Cali, así como su división política administrativa y malla vial.

Las variables incluidas en el análisis fueron: municipio donde ocurrió la defunción, sitio de la muerte (hospital/clínica, centro/puesto de salud, casa/domicilio, lugar de trabajo, vía pública, otro sitio), hora del día, día de la semana, mes, sexo, grupo de edad, año de 
registro de la defunción, condición de la víctima (motociclista, ciclista, conductor, peatón, ocupante), dirección de ocurrencia, código de causa básica de defunción y población total por año.

A los registros de defunción que contaban con la dirección de ocurrencia del accidente que causó la muerte, la nomenclatura vial se normalizó y depuró para luego geo-codificar. La nomenclatura con información parcial se revisó, se normalizó hasta donde fue posible y después se geo-codificó. Se utilizó la base geográfica (malla vial) de Cali a escala 1:5.000 del Departamento Administrativo Nacional de Estadística (DANE) construida para el Censo 2005, actualizada por los autores de este estudio, con el sistema de coordenadas MAGNA-SIRGAS de Cali de 2009 (Alcaldia de Santiago de Cali, 2018).

Las muertes debidas a lesiones de tránsito fueron analizadas en términos de frecuencias absolutas y relativas. Se consideraron grupos quinquenales de edad. Se calcularon tasas crudas y ajustadas por edad usando el método directo que tuvo en cuenta como población de referencia la población mundial de la OMS (Ahmad et al., 2001). Para representar gráficamente el número de casos de mortalidad promedio y las tasas promedio del periodo, se utilizó una representación cartográfica en coropletas con intervalos de cuartiles, donde el cuartil más alto representó el más alto número de casos y las tasas más altas por comuna.

Para el análisis espacial se utilizó la estimación de la densidad de Kernel que detectó aquellas áreas en las vías en las que existió una mayor densidad de muertes (Silverman, 1986). Para el análisis de la estimación de la densidad de Kernel, se consideró un tamaño de píxel de 5,8 × 5,8 metros que cubría el área urbana de Cali, mediante una malla organizada en 2.419 columnas y 3.691 filas, lo que permitió una visualización adecuada a una escala aproximada de 1:10.000; siendo una escala ideal para un análisis del conjunto de elementos. Se aplicó la estimación de la densidad de Kernel a todos los casos que se presentaron durante el periodo 2008-2013; también se comparó el comportamiento de este valor año tras año, para obtener un valor de crecimiento o decrecimiento de la densidad y comprobar la existencia de un estacionamiento o movilidad espacial de la mortalidad. También la estimación de la densidad de Kernel se empleó por tipo de actor vial.

Los datos obtenidos se procesaron en hojas de cálculo de Microsoft Excel 2013. La información se analizó con el programa estadístico Stata versión 12 y los datos espaciales con el programa ArcGis versión 10.2.2 (módulo de análisis espacial). La geo-codificación se hizo a partir de la dirección de la vía donde ocurrió la muerte con Google Earth. De acuerdo con el artículo 11 de la resolución 8430 de 1993 del Ministerio de Salud de Colombia, este estudio se clasificó "sin riesgo".

\section{RESULTADOS}

Entre 2008 y 2013, en Cali, se registraron 1.857 muertes debidas a lesiones de tránsito. Los fallecimientos predominaron en hombres, con un promedio de 310 muertes por año. El grupo de edad con la mayor proporción de muertes fue el de 20 a 29 años de edad. Durante los fines de semana, sábado y domingo, se presentaron más muertes. El mes con la mayor frecuencia de fallecimientos fue diciembre. De acuerdo con la condición de la víctima, fallecieronmás motociclistas y peatones (Cuadro 2). 


\section{Cuadro 2.}

Características de los fallecidos en lesiones de tránsito. Cali, 2003 - 2013

\begin{tabular}{|c|c|c|}
\hline Sexo & n. & $\%$ \\
\hline Hombre & 1.485 & 80,0 \\
\hline Mujer & 372 & 20,0 \\
\hline Grupo de edad & n. & $\%$ \\
\hline 0 a 9 & 22 & 1,2 \\
\hline 10 a 19 & 117 & 6,3 \\
\hline 20 a 29 & 411 & 22,1 \\
\hline 30 a 39 & 270 & 14,5 \\
\hline 40 a 49 & 266 & 14,3 \\
\hline 50 a 59 & 232 & 12,5 \\
\hline 60 a 69 & 210 & 11,3 \\
\hline 70 a 79 & 200 & 10,8 \\
\hline 80 y más & 127 & 6,8 \\
\hline Día & n. & $\%$ \\
\hline Lunes & 259 & 13,9 \\
\hline Martes & 218 & 11,7 \\
\hline Miércoles & 251 & 13,5 \\
\hline Jueves & 246 & 13,2 \\
\hline Viernes & 228 & 12,3 \\
\hline Sábado & 318 & 17,1 \\
\hline Domingo & 337 & 18,1 \\
\hline
\end{tabular}




\section{Cuadro 2.}

Características de los fallecidos en lesiones de tránsito. Cali, 2003 - 2013

\begin{tabular}{|c|c|c|}
\hline Mes & n. & $\%$ \\
\hline Enero & 143 & 7,7 \\
\hline Febrero & 144 & 7,8 \\
\hline Marzo & 150 & 8,1 \\
\hline Abril & 160 & 8,6 \\
\hline Mayo & 144 & 7,8 \\
\hline Junio & 158 & 8,5 \\
\hline Julio & 162 & 8,7 \\
\hline Agosto & 181 & 9,7 \\
\hline Septiembre & 142 & 7,6 \\
\hline Octubre & 140 & 17,5 \\
\hline Noviembre & 155 & 8,3 \\
\hline Diciembre & 178 & 9,6 \\
\hline Víctima & n. & $\%$ \\
\hline Ciclista & 220 & 11,8 \\
\hline Conductor & 23 & 1,2 \\
\hline Motociclista & 708 & 38,1 \\
\hline Ocupante & 37 & 2,0 \\
\hline Pasajero & 41 & 2,2 \\
\hline Peatón & 695 & 37,4 \\
\hline Persona lesionada & 133 & 7,2 \\
\hline
\end{tabular}

Fuente: Elaboración de los autores partir de Estadísticas vitales, DANE 
De 609 muertes que ocurrieron en la vía pública, 431 (70,8\%) contaban con la hora de la defunción. Entre las seis de la tarde y la media noche se registraron 119 (27,6\%) fallecimientos, seguidas por las que ocurrieron entre el mediodía y antes de las seis de la tarde $(116,26,9 \%)$.

La tasa promedio de mortalidad para el periodo fue de 13,6 por 100.000 habitantes. La tendencia en las tasas de mortalidad debida a lesiones de tránsito tuvo una pendiente descendente (Figura 1). La tendencia de las tasas de mortalidad ajustadas por edad de motociclistas descendió entre 2009 y 2011 para luego permanecer estable; las de ocupantes disminuyeron levemente, mientras que las de peatones, ciclistas y otros mantuvieron un patrón estable (Figura 2).

Figura 1. Mortalidad por lesiones de tránsito. Cali, Colombia. 2008 - 2013.

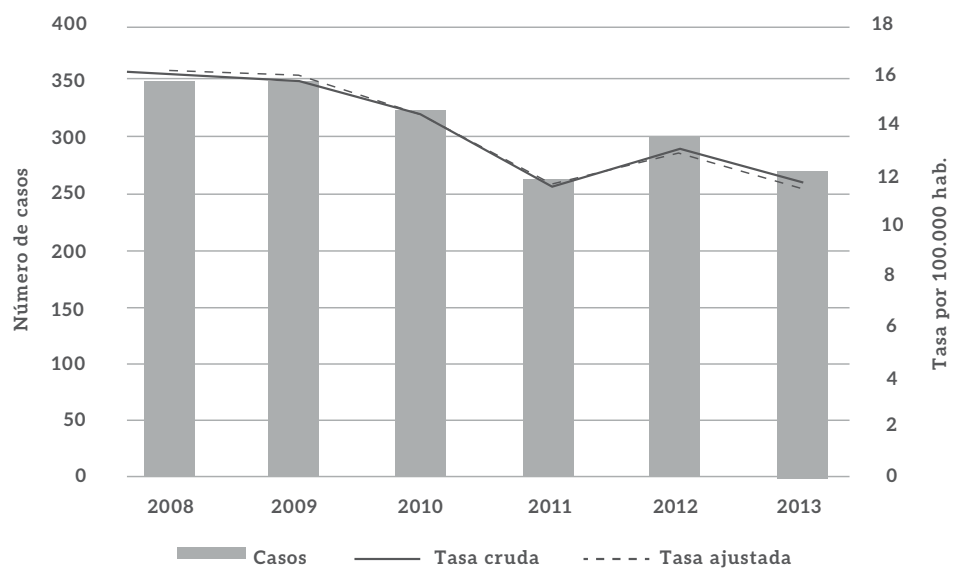

Fuente: Elaboración de los autores partir de Estadísticas vitales, DANE

Figura 2. Tasas ajustadas por edad de mortalidad debida a lesiones de tránsito según condición de la víctima. Cali, Colombia. 2008 - 2013

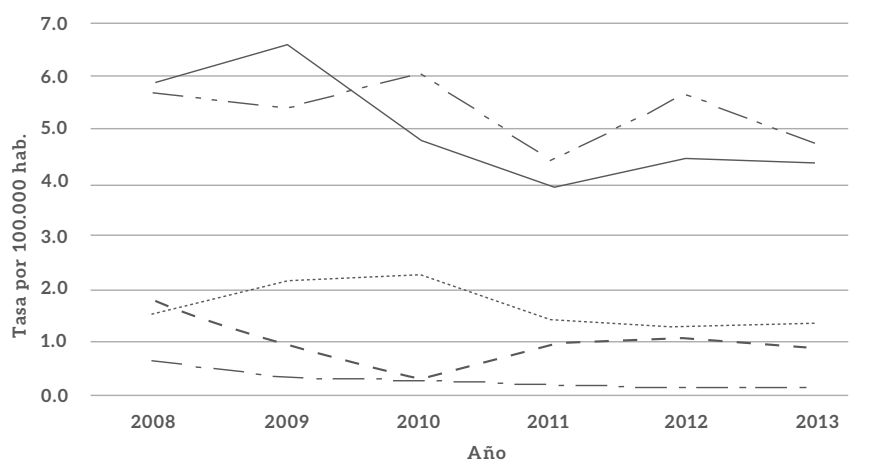

Motociclista - - Peatón

Ciclista - - Otro - - Ocupante

Fuente: Elaboración de los autores partir de Estadísticas vitales, DANE 
Dentro de la ciudad, las comunas que reportaron el mayor número de muertes fueron la 19 (123, 6.6\%) ubicada al occidente, la 8 (115, 6.2\%) localizada en el nororiente y la 3 (92, $5.0 \%)$ establecida en el centro. Las tasas más altas se observaron para las comunas 22 (49,0 muertes por 100.000 habitantes), localizada en el sur del municipio, 3 (33,4 por 100.000) y 9 (32,1 por 100.000) en el centro de la ciudad (Figura 3). Las muertes se distribuyeron a lo largo de las vías principales (Figura 4).

\section{Cuadro 3.}

Mortalidad debida a lesiones de tránsito por comunas. Cali 2008 - 2013

\begin{tabular}{|c|c|c|c|}
\hline Comuna & Muertes & $\%$ & Tasa \\
\hline Comuna 1 & 12 & 0,6 & 2,63 \\
\hline Comuna 2 & 81 & 4,4 & 12,68 \\
\hline Comuna 3 & 92 & 5,0 & 33,41 \\
\hline Comuna 4 & 67 & 3,6 & 20,36 \\
\hline Comuna 5 & 52 & 2,8 & 8,04 \\
\hline Comuna 6 & 32 & 1,7 & 2,93 \\
\hline Comuna 7 & 80 & 4,3 & 18,10 \\
\hline Comuna 8 & 115 & 6,2 & 18,88 \\
\hline Comuna 9 & 90 & 4,8 & 32,14 \\
\hline Comuna 10 & 72 & 3,9 & 11,02 \\
\hline Comuna 11 & 63 & 3,4 & 10,04 \\
\hline Comuna 12 & 54 & 2,9 & 13,30 \\
\hline Comuna 13 & 80 & 4,3 & 7,58 \\
\hline Comuna 14 & 72 & 3,9 & 7,30 \\
\hline Comuna 15 & 66 & 3,6 & 7,58 \\
\hline Comuna 16 & 50 & 2,7 & 8,13 \\
\hline Comuna 17 & 72 & 3,9 & 9,58 \\
\hline Comuna 18 & 18 & 1,0 & 2,60 \\
\hline Comuna 19 & 123 & 6,6 & 18,87 \\
\hline Comuna 20 & 10 & 0,5 & 2,46 \\
\hline Comuna 21 & 37 & 2,0 & 6,01 \\
\hline Comuna 22 & 29 & 1,6 & 49,01 \\
\hline
\end{tabular}

Fuente: Elaboración de los autores partir de Estadísticas vitales, DANE 
Figura 3. Número promedio de muertes y tasas promedio de mortalidad debida a lesiones de tránsito por comunas. Cali, Colombia. 2008 - 2013
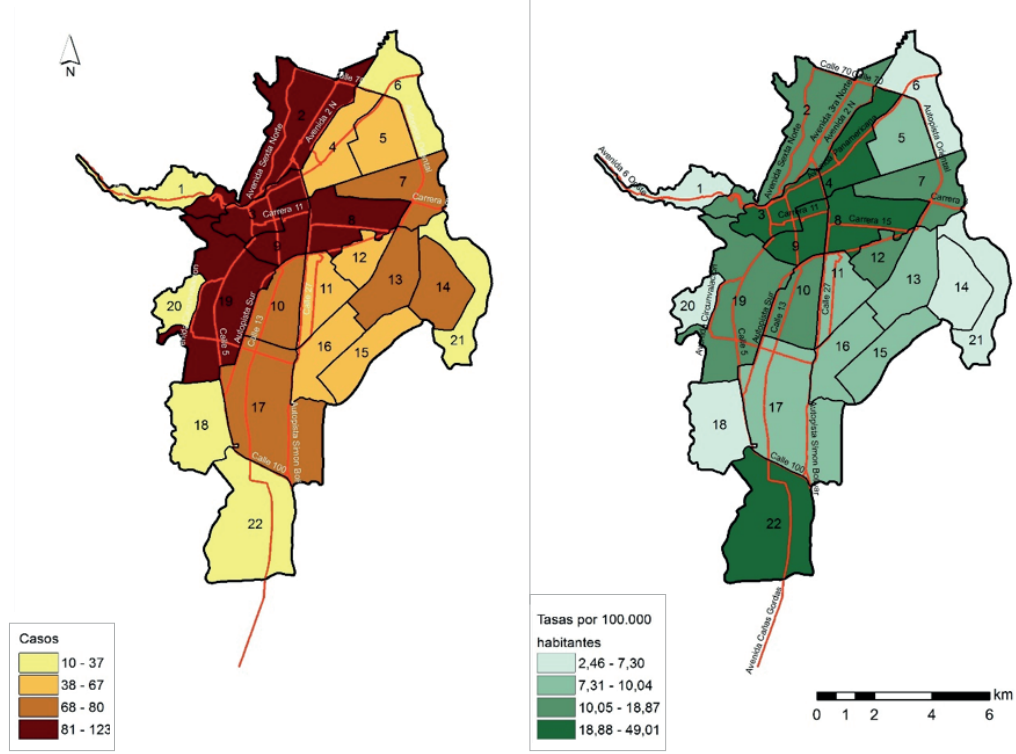

Fuente: Elaboración de los autores partir de Estadísticas vitales, DANE

Figura 4. Distribución de muertes por accidente de tránsito.

(A) distribución puntual; (B) densidad kernel. Cali, Colombia. 2008 - 2013
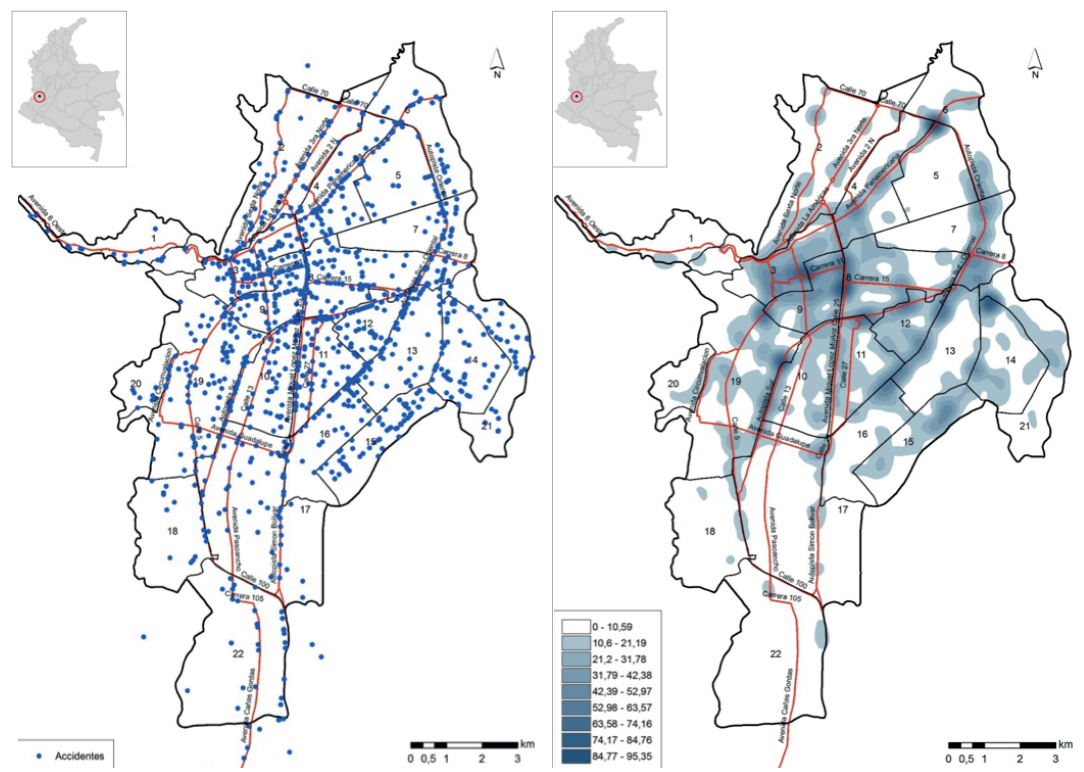

Fuente: Elaboración de los autores a partir de las Estadísticas Vitales - DANE 
Se geo-referenciaron 1.756 (94,6\%) muertes, el 5,4\% restante se omitió por falta de información. La estimación de la densidad de Kernel mostró distintas áreas con una alta densidad de muertes (color azul intenso en los mapas), localizadas principalmente en la confluencia de la comuna 3 con la comuna 9 y de la comuna 8 con la comuna 9; en la intersección de la comuna 4 con las comunas 5 y 6, en el extremo norte de la comuna 14 y en la confluencia de la comuna 19 con la comuna 10, zonas en las que la densidad de muertes fue de 85 y más por km2 (Figura 5).

La tendencia del comportamiento espacial anual mostró una reducción paulatina en la densidad focal; entre 2008 y 2011 presentó áreas en las que la densidad de muertes fue de 23 y más por km2, para luego aumentar y en 2013 mostrar áreas con una densidad de muertes que estuvo alrededor de 55 por km2 (la mayor densidad de muertes por lesiones de tránsito) (Figura 6).

Figura 6. Estimador de la densidad de Kernel de la mortalidad debida a lesiones de tránsito por año. Cali, 2008 - 2013.

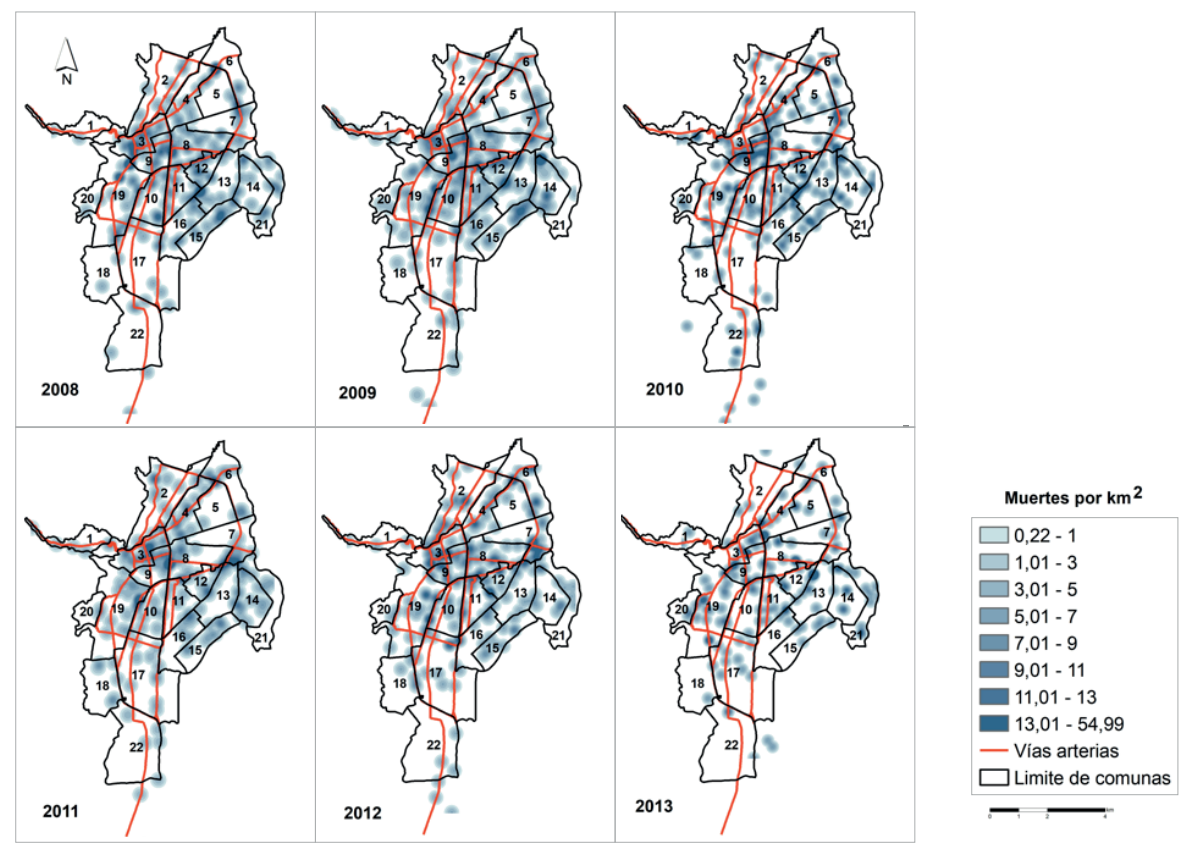

Fuente: Elaboración de los autores partir de Estadísticas vitales, DANE

De acuerdo con el actor vial fallecido, la estimación de la densidad de Kernel mostró áreas en las que la densidad de muertes para: motociclistas fue de 27 y más por $\mathrm{km} 2$, sitios que se localizaron principalmente en las comuna 9, 7,13,14, 15 y 19; peatones fue de 53 y más por km2, lugares que se situaron en las comuna 3, 9 y 14; ciclistas fue de 33 y más por km2; y ocupante de vehículo fue de 23 y más muertes por km2 (figura 7). 
Figura 7. Estimador de la densidad de Kernel de la mortalidad debida a lesiones de tránsito según actor vial. Cali, 2008 - 2013

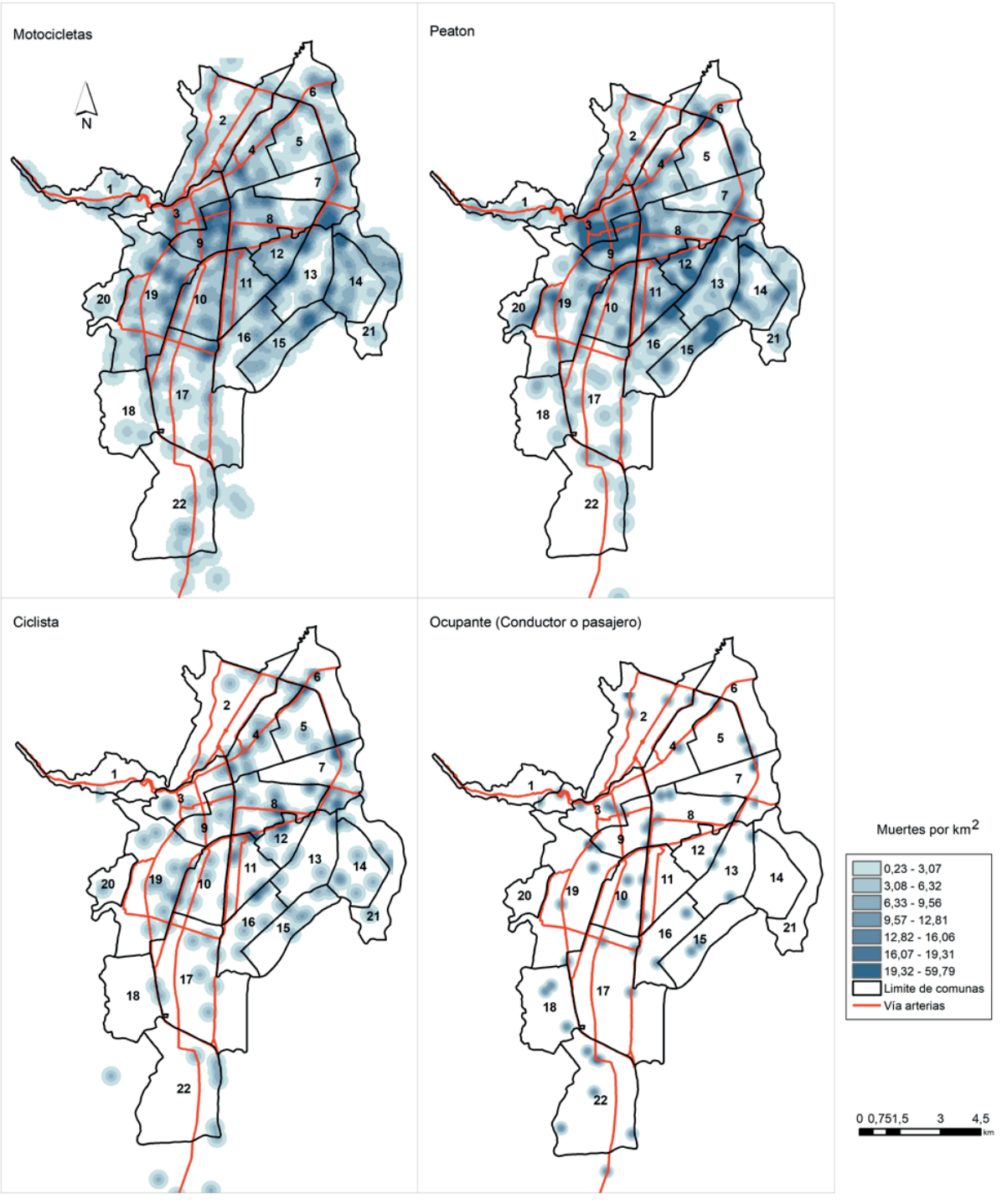

Fuente: Elaboración de los autores partir de Estadísticas vitales, DANE 


\section{ANÁLISIS Y DISCUSIÓN}

En Cali, las lesiones de tránsito son responsables de un importante número de muertes, registrandose entre 2008 y 20131.875 muertes. Una frecuencia alta de defunciones se presentó en las comunas 3, 8, 9 y 19, caracterizadas por la alta concentración de comercio, actividad industrial y alto grado de informalidad. La mayor parte de las muertes se presentaron entre motociclistas y peatones. La tasa promedio de mortalidad para el periodo fue de 13,6 fallecimientos por 100.000 habitantes. Temporal y espacialmente, la mortalidad evidenció disminución durante el periodo estudiado y se identificaron agrupamientos en distintas comunas del municipio.

La mayoría de víctimas mortales consecuencia de las lesiones de tránsito fueron hombres, hecho consistente con estudios previos, y que se puede ser debido a que ellos conducen más kilómetros que las mujeres, y las lesiones en los que se involucran son más graves (Li, Baker, Langlois, \& Kelen, 1998). Además, se puede sumar la menor percepción de riesgo y la mayor agresividad al conducir que tienen los hombres jóvenes que los hace tomar comportamientos peligrosos (Meneses Falcón, Gil García, \& Romo Avilés, 2010).

El mayor número de muertes se presentó en el grupo de edad de 20 a 29 años, como se ha evidenciado en otras investigaciones (Hamzeh et al., 2016; Hidalgo-Solórzano, Híjar, Mora-Flores, Treviño-Siller, \& Inclán-Valadez, 2008; Jooma \& Shaikh, 2016; Ziyab \& Akhtar, 2012) y que podría ser debido a que en este grupo poblacional falta de experiencia para conducir, adoptan comportamientos inseguros como exceso de velocidad y consumo de bebidas alcohólicas (Orsi et al., 2012), y la falta de conocimiento de las normas de seguridad vial, tanto por conductores (Bates, Davey, Watson, King, \& Armstrong, 2014) como por peatones (Alex Echeverry, Mera, Villota, \& Zárate, 2005). Al igual que en otras ciudades, los fines de semana se presentan más muertes, esto puede ser debido a que ante un mayor tiempo para el ocio se presenta consumo de alcohol, se abusa de la velocidad que lleva a lesiones de tránsito más graves (W Odero, P Garner, \& A Zwi, 1997). Similar a lo observado en Vietnam (Ngo et al., 2012), el más alto número de muertes ocurrió en diciembre, mes en el que se el país se realizan distintas celebraciones (velitas, navidad, fin de año) que hacen que aumente el tránsito de peatones, se incremente el uso de vehículos (Ngo et al., 2012) y no se respeten las normas de tránsito (Derry, 2017).

Más de una cuarta parte de los fallecimientos se presentó entre las seis de la tarde y la media noche. Se ha observado que, en la noche, generalmente ocurren las lesiones más aparatosas. Posiblemente sea debido a largos periodos de conducción que sin descanso adecuado pueden ocasionar cansancio y somnolencia (Orsi et al., 2012), falta de respeto por las señales de tránsito, exceso de velocidad y consumo de alcohol (Derry, 2017). Adicionalmente, la iluminación débil y las precarias condiciones de las vías públicas pueden ser factores que contribuyeron a la ocurrencia de lesiones. Por otra parte, las lesiones de tráfico en este horario se podrían deber a que los conductores no ajustan su velocidad a la visibilidad reducida en condiciones de oscuridad (Jägerbrand \& Sjöbergh, 2016).

La mayor parte de las víctimas correspondió a motociclistas y peatones. En los últimos años en Cali como en otras ciudades colombianas aumentó el número de usuarios de motocicletas. Esta situación está favorecida por su bajo costo de adquisición y uso, ser una alternativa de transporte ante la congestión vehicular debido a que reduce los tiempos de viaje, tener un reducido control y regulación (Rodríguez, Santana, \& Pardo, 
2015). La falta de infraestructura adecuada como puentes peatonales, pasos deprimidos, cebras o pasos para peatones, aunada a una deficiente educación sobre seguridad vial pudo influir en los peatones (Kareem, 2003; Ngo et al., 2012; Ramage-Morin, 2008).

La tendencia en la mortalidad debida a lesiones de tránsito fue descendente, al igual que en Irán (Yousefzadeh-Chabok, Ranjbar-Taklimie, Malekpouri, \& Razzaghi, 2016), probablemente resultado de las distintas medidas y políticas adoptadas e implementadas respecto del tránsito (Melchor et al., 2015; Novoa et al., 2009; Orsi et al., 2012; Patel et al., 2016). En particular, en Cali, distintas medidas de control y prevención se han implementado tales como programas de educación en seguridad vial dirigidos a conductores, cumplimiento en el uso de cinturones de seguridad y cascos de seguridad en motociclistas, control de la velocidad y del consumo de alcohol al conducir (Cuadros, 2016), establecimiento de cámaras de foto detección (González \& Prada, 2016), aumento de guardas de tránsito (Liscano \& Montoya, 2014), implementación del uso de un carril para movilización y prohibición del zig-zageo para los motociclistas (Forero \& Rodas, 2016), han mostrado cierto impacto en el comportamiento de la tendencia de la mortalidad (Kareem, 2003; Ngo et al., 2012; Ramage-Morin, 2008). Además, la reducción en la tendencia de la mortalidad, en parte puede deberse a la mejora de la red de vías y a una mejor atención de salud (Ziyab \& Akhtar, 2012).

La estimación de la densidad de Kernel se ha utilizado para identificar áreas de riesgo y los "puntos calientes" de las lesiones de tráfico (Anderson, 2009; Jägerbrand \& Sjöbergh, 2016; Xie \& Yan, 2013). Este análisis espacial indicó que las áreas de mayor densidad de muertes se localizaron en las intersecciones viales y en los tramos de las avenidas principales, como la autopista Sur-Oriental y la calle 5 entre carreras 5 y 10 entre otras. En esas zonas densamente pobladas, por lo general confluye un alto número de peatones y una cantidad considerable de vehículos. La mortalidad debida a lesiones de tránsito en esas áreas puede ser el resultado de una falta de observación a las normas de tránsito aunada a una débil infraestructura vial (Almeida, Pignatti, \& Espinosa, 2009; Patel et al., 2016). Las diferencias en la mortalidad entre comunas posiblemente sea debida a la alta densidad de peatones y tráfico vehicular, la mayor velocidad y temeridad del conductor, patrones de tráfico complejos (de Andrade et al., 2014), las vías más grandes (Patel et al., 2016) y deficiencias en la iluminación. Como en Brasil (Libânio, Neto, \& Maria, 2012), la identificación de las áreas de mayor densidad permiten conocer las áreas geográficas con mayor riesgo de muertes debidas a accidente de tránsito, el actor vial implicado, así como los cambios en los patrones espaciales en el tiempo para establecer o ratificar las medidas pertinentes para reducir y prevenir la mortalidad.

Entre las limitaciones de este análisis se deben mencionar las derivadas de la fuente de información y de la metodología geoespacial empleadas. Respecto de la fuente de información, los certificados de defunción pueden no tener datos sobre: el tipo de vehículo implicado en el accidente (automóvil, bicicleta, motocicleta u otro) y la dirección del lugar de ocurrencia del hecho. En relación con esta variable, los datos para la georreferenciación no estuvieron disponibles para el 5,4\% de los fallecimientos que ocurrieron durante el periodo 2008-2013. En relación con la estimación de la densidad de Kernel se ha observado que está sometida a la designación que haga el investigador del ancho de banda de búsqueda (Gómez-Barroso, López-Cuadrado, Llácer, Palmera Suárez, \& Fernández-Cuenca, 2015). Para este análisis, se seleccionó un ancho de banda de $1 \mathrm{~km}$ (1.000 m), y se calculó la densidad de Kernel por km2/año para cada una de las celdas en que fue dividido el mapa de Cali. 
El conocimiento de la distribución de las lesiones de tránsito dentro de la ciudad, junto con las defunciones que de ellos se deriva, permite tener información relevante para la toma de decisiones respecto de su prevención. Mejora en la infraestructura vial, campañas de seguridad vial y programas educativos son medidas que contribuyen con la reducción prevención de las lesiones de tráfico.

\section{CONCLUSIONES}

La mortalidad debida a lesiones de tránsito, por lo general ocurrió en áreas con un alto flujo de individuos y de vehículos. Este análisis ha identificado las características de las víctimas mortales que dejan las lesiones de tránsito y su distribución temporal y espacial. En Cali para reducir la mortalidad derivada de las lesiones de tránsito, los resultados presentados pueden contribuir con el apoyo del trabajo en prevención de las lesiones y de la mortalidad, así como de la promoción de la seguridad vial desde distintos sectores sociales y en particular sobre los actores viales que pueden estar involucrados.

\section{REFERENCIAS BIBLIOGRÁFICAS}

Ahmad, O. B., Boschi-Pinto, C., Lopez, A. D., Murray, C. J. L., Lozano, R., \& Inoue, M. (2001). Age standardization of rates: a new WHO standard. GPE Discussion Paper Series. https://doi. org/10.1161/HYPERTENSIONAHA.114.04394

Alcaldia de Santiago de Cali. (2018). Proyecciones y transformaciones para ArcGIS. Retrieved February 28, 2018, from http://www.cali.gov.co/planeacion/publicaciones/105290/ proyecciones_transformaciones_arcgis_idesc/

Almeida, L. V. de C., Pignatti, M. G., \& Espinosa, M. M. (2009). Principais fatores associados à ocorrência de acidentes de trânsito na BR 163, Mato Grosso, Brasil, 2004. Cadernos de Saúde Pública, 25(2), 303-312. https://doi.org/10.1590/s0102-311x2009000200008

Anderson, T. K. (2009). Kernel density estimation and K-means clustering to profile road accident hotspots. Accident Analysis and Prevention, 41(3), 359-364. https://doi.org/10.1016/j. aap.2008.12.014

Bates, L. J., Davey, J., Watson, B., King, M. J., \& Armstrong, K. (2014). Factors contributing to crashes among young drivers. Sultan Qaboos University Medical Journal, 14(3), 297-305. https:// doi.org/10.1182/blood-2009-09-240440

Colombia. Contraloría General de la República. (2012). Seguridad Vial en Colombia. Especial seguimiento a los resultados de la política pública.

Colombia. Contraloría General de la República. (2014). Política pública de Seguridad Vial. Retrieved from https://www.contraloria.gov.co/documents/20181/465902/18+Seguridad+Vial.pdf/ a89624ec-00e4-48ce-9a72-188b278a2b69?version=1.0

Colombia, M. (2015). Plan Nacional de Seguridad Vial 2011 - 2021 (Segunda ed). Bogotá D.C. Retrieved from https://www.mintransporte.gov.co/publicaciones/3239/plan-nacional-de-seguridad-vial/

Cuadros, F. N. (2016). La política de seguridad vial y la incidencia en la mortalidad vial en el Valle del Cauca en el periodo 2012-2015. Un estudio de caso. Universidad del Valle. Retrieved from http://bibliotecadigital.univalle.edu.co/bitstream/10893/9977/1/CB-0542792.pdf 
Cuervo, Z. (2016, March 27). Bajan los accidentes de tránsito en Cali este año, pero aumentan los muertos. El País.Com.Co. Retrieved from https://www.elpais.com.co/cali/bajan-losaccidentes-de-transito-en-este-ano-pero-aumentan-los-muertos.html

de Andrade, L., Vissoci, J. R. N., Rodrigues, C. G., Finato, K., Carvalho, E., Pietrobon, R., ... de Barros Carvalho, M. D. (2014). Brazilian Road Traffic Fatalities: A Spatial and Environmental Analysis. PLoS ONE, 9(1), e87244. https://doi.org/10.1371/journal.pone.0087244

Derry, J. D. (2017). the Prevalence of Alcohol Use Among Road Users and Its Impact on Traffic Crash Severity in. Queensland University of Technology. Retrieved from https://eprints. qut.edu.au/112415/1/James_Damsere Derry_Thesis.pdf

Dummer, T. J. B. (2008). Commentary Social and spatial inequalities in health Concerns about social and spatial polarization-especially with socio-economic inequalities, inequities and poverty. Cmaj, 178(9), 7-10. https://doi.org/10.1503/cmaj.071783

Echeverry, A., Mera, J. J., Villota, J., \& Zárate, L. C. (2005). Actitudes y comportamientos de los peatones en los sitios de alta accidentalidad en Cali. Colombia Médica, 36(2), 79-84.

Echeverry, A., Mera, J., Villota, J., \& Zárate, L. (2005). Actitudes y comportamientos de los peatones en los sitios de alta accidentalidad en Cali. Colombia Medica, 36, 79-84. Retrieved from http://colombiamedica.univalle.edu.co/index.php/comedica/article/view/336/1116

Forero, J. C., \& Rodas, J. C. (2016). Modelación de estrategias de manejo de carriles para disminución de congestión y accidentalidad vial, Plan Piloto Autopista Sur-Oriental. Pontificia Universidad Javeriana - Cali. Retrieved from http://vitela.javerianacali.edu.co/bitstream/ handle/11522/7411/Modelacion_estrategias_manejo.pdf?sequence=1

Gómez-Barroso, D., López-Cuadrado, T., Llácer, A., Palmera Suárez, R., \& Fernández-Cuenca, R. (2015). Análisis espacial de los accidentesas lesiones de tráfico con-víctimas mortales en carretera en España, 2008-2011. Gaceta Sanitaria, 29(S1), 24-29. https://doi. org/10.1016/j.gaceta.2015.02.009

González, J. F., \& Prada, S. I. (2016). Cámaras de fotodetección y accidentalidad vial. Evidencia para la ciudad de Cali. Revista Desarrollo y Sociedad, (77), 131-182. https://doi.org/10.13043/ dys.77.4

Hamzeh, B., Najafi, F., Karamimatin, B., Ahmadijouybari, T., Salari, A., \& Moradinazar, M. (2016). Epidemiology of traffic crash mortality in west of Iran in a 9 year period. Chinese Journal of Traumatology - English Edition, 19(2), 70-74. https://doi.org/10.1016/j.cjtee.2015.12.007

Hidalgo-Solórzano, E., Híjar, M., Mora-Flores, G., Treviño-Siller, S., \& Inclán-Valadez, C. (2008). Accidentes de tránsito de vehículos de motor en la población joven: evaluación de una intervención educativa en Cuernavaca, Morelos. Salud Publica Mex, 50(50 supl), 560-568.

Instituto Nacional de Medicina Legal y Ciencias Forenses. (2015). Forensis 2014. Datos para la vida (Primera ed). Bogotá D.C.: Imprenta Nacional. Retrieved from http://www.medicinalegal. gov.co/documents/20143/49520/Forensis+2014+Datos+para+la+vida.pdf

Instituto Nacional de Medicina Legal y Ciencias Forenses. (2016). Forensis 2015. Datos para la vida (Primera ed). Bogotá D. C.: Imprenta Nacional. Retrieved from http://www.medicinalegal. gov.co/documents/20143/49523/Preliminares.pdf

Jägerbrand, A. K., \& Sjöbergh, J. (2016). Effects of weather conditions, light conditions, and road lighting on vehicle speed. SpringerPlus, 5(1). https://doi.org/10.1186/s40064-016-2124-6 
Jooma, R., \& Shaikh, M. A. (2016). Descriptive epidemiology of karachi road traffic crash mortality from 2007 to 2014. Journal of the Pakistan Medical Association, 66(11), 1475-1480.

Kareem, A. (2003). Review of Global Menace of Road Accidents With Special Reference To Malaysia-a Social Perspective. Malaysian Journal of Medical Sciences, 10(2), 31-39. Retrieved from https://www.ncbi.nlm.nih.gov/pmc/articles/PMC3561885/pdf/mjms-10-2031.pdf

Li, G., Baker, S. P., Langlois, J. A., \& Kelen, G. D. (1998). Are female drivers safer? An application of the decomposition method. Epidemiology, 9(4), 379-384. https://doi.org/10.1097/00001648199807000-00006

Libânio, O., Neto, D. M., \& Maria, M. (2012). Mortalidade por Acidentes de Transporte Terrestre no Brasil na última década : tendência e aglomerados de risco Mortality due to Road Traffic Accidents in Brazil in the last decade : trends and risk clusters. Ciência \& Saúde Coletiva, 17(9), 2223-2236.

Liscano, T. A., \& Montoya, D. (2014). Mejoramiento de la movilidad y el tránsito en la ciudad de Santiago de Cali a través de la planeación y diseño de dos servicios basados en TIC. ICESI. Retrieved from https://repository.icesi.edu.co/biblioteca_digital/bitstream/ 10906/77787/3/liscano_mejoramiento_movilidad_2014.pdf

Melchor, I., Nolasco, A., Moncho, J., Quesada, J. A., Pereyra-Zamora, P., García-Senchermés, C., ... Salinas, M. (2015). Trends in mortality due to motor vehicle traffic accident injuries between 1987 and 2011 in a Spanish region (Comunitat Valenciana). Accident Analysis and Prevention, 77, 21-28. https://doi.org/10.1016/j.aap.2015.01.023

Meneses Falcón, C., Gil García, E., \& Romo Avilés, N. (2010). Adolescentes, situaciones de riesgo y seguridad vial. Atención Primaria, 42(9), 452-458. https://doi.org/10.1016/j.aprim. 2009.10.013

Naciones Unidas. Resolucion 64/255 Mejoramiento de la Seguridad Vial, Naciones Unidas § (2010). Retrieved from http://www.who.int/violence_injury_prevention/publications/road_ traffic/UN_GA_resolution-54-255-en.pdf

Ngo, A. D., Rao, C., Phuong Hoa, N., Hoy, D. G., Thi Quynh Trang, K., \& Hill, P. S. (2012). Road traffic related mortality in Vietnam: Evidence for policy from a national sample mortality surveillance system. BMC Public Health, 12(1), 1. https://doi.org/10.1186/1471-2458-12-561

Novoa, A. M., Santamarina-Rubio, E., Mari-Dell'Olmo, M., Perez, K., Ferrando, J., Zori, P., ... Borrell, C. (2009). Road safety in the political agenda: the impact on road traffic injuries. Journal of Epidemiology \& Community Health, 65(3), 218-225. https://doi.org/10.1136/ jech.2009.094029

Olaya, R. A. (2015). Modelo espacial de muertes por accidentes de tránsito en la zona urbana de Cali, Colombia durante el periodo 2004-2014. Universidad de Manizales.

Organización Panamericana de la Salud. (1995). Clasificación Estadística Internacional de Enfermedades y Problemas Relacionados con la Salud. Décima Revisión (Ed. 2003). Washington, D.C.: Organización Panamericana de la Salud.

Orsi, C., Bertuccio, P., Morandi, A., Levi, F., Bosetti, C., \& Vecchia, C. La. (2012). Trends in motor vehicle crash mortality in Europe, 1980-2007. Safety Science, 50(4), 1009-1018. https://doi. org/10.1016/j.ssci.2011.12.008

Patel, A., Krebs, E., Andrade, L., Rulisa, S., Vissoci, J. R. N., \& Staton, C. A. (2016). The epidemiology of road traffic injury hotspots in Kigali, Rwanda from police data. BMC Public Health, 16(1), 1-10. https://doi.org/10.1186/s12889-016-3359-4 
Programa de las Naciones Unidas para el desarrollo. (2015). Objetivos de Desarrollo Sostenible. Retrieved March 7, 2019, from http://www.who.int/topics/sustainable-developmentgoals/targets/es/

Ramage-Morin, P. L. (2008). Motor vehicle accident deaths, 1979 to 2004. Health Reports / Statistics Canada, Canadian Centre for Health Information = Rapports Sur La Santé / Statistique Canada, Centre Canadien d'information Sur La Santé, 19(3), 45-51.

Reyes-Ortiz, C. A., Espitia, V. E., Vélez, L. F., \& Espinoza, R. (1998). Lesiones fatales ocasionadas por vehículo motor a personas mayores de 60 años en Cali, 1993-1997. Colombia Medica (Vol. 29).

Rodríguez, D. A., Santana, M., \& Pardo, C. F. (2015). La motocicleta en América Latina. Caracterización de su uso en impactos en la movilidad en cinco ciudades de la Región. Mycological Research (Vol. 111). Bogotá D.C.: Despacio. Retrieved from http://scioteca.caf.com/ bitstream/handle/123456789/754/CAF LIBRO motos digital.pdf

Sánchez, Á. I., Suffoleto, B., Mena, J., Gutiérrez, M. I., \& Puyana, J.-C. (2014). The association between alcohol restriction policies and vehicle-related mortality in Cali, Colombia, 1998-2008. The International Journal of Alcohol and Drug Research, 3(2), 149. https://doi.org/10.7895/ ijadr.v3i2.157

Silverman, B. W. (1986). Density Estimation for Statistics and Data Analysis (p. 22). London: Chapman and Hall. https://doi.org/10.1177/030098589603300105

W Odero, P Garner, \& A Zwi. (1997). Road traffic injuries in developing countries: a comprehensive review of epidemiological studies. Tropical Medicine and International Health, 2(5), 445-460.

World Health Organization. (2015). Deaths on the roads. Retrieved January 26, 2016, from https:// extranet.who.int/roadsafety/death-on-the-roads/

World Health Organization. (2013). Global status report on road safety 2013: supporting a decade of action., 1, 318. https://doi.org/10.1258/jrsm.2010.090426

Xie, Z., \& Yan, J. (2013). Detecting traffic accident clusters with network kernel density estimation and local spatial statistics: An integrated approach. Journal of Transport Geography, 31, 64-71. https://doi.org/10.1016/j.jtrangeo.2013.05.009

Yousefzadeh-Chabok, S., Ranjbar-Taklimie, F., Malekpouri, R., \& Razzaghi, A. (2016). A Time Series Model for Assessing the Trend and Forecasting the Road Traffic Accident Mortality. Archives of Trauma Research, 5(3). https://doi.org/10.5812/atr.36570

Ziyab, A. H., \& Akhtar, S. (2012). Incidence and trend of road traffic injuries and related deaths in Kuwait: 2000-2009. Injury, 43(12), 2018-2022. https://doi.org/10.1016/j.injury.2011.09.023 